\title{
Visualization and Spectral Analysis of Pubic Periosteum Atrophy
}

\author{
Vladimir I. Belokonev ${ }^{1}$, Valery P. Zakharov ${ }^{2 *}$, Sergey Y. Pushkin ${ }^{1}$, Zinaida V. Kovaleva ${ }^{1}$, \\ Andrey V. Zharov' ${ }^{1}$, Natalya S. Burnaeva ${ }^{1}$, Elizaveta V. Zakharova ${ }^{1}$, and Yulia A. Khristoforova ${ }^{2}$ \\ ${ }^{1}$ Samara State Medical University, 89 Chapayevskaya str., Samara 443099, Russia \\ ${ }^{2}$ Samara National Research University, 34 Moskovskoe shosse, Samara 443086, Russia \\ *e-mail: zakharov@ssau.ru
}

\begin{abstract}
The atrophy of the pubic periosteum is developing in patients with a long history of femoral hernia. The traditional plastic surgery methods are efficient only in patients with femoral hernia when the Cooper ligament of the pubic periosteum is preserved. The aim of this study is to determine the thinning and atrophy degree of the pubic periosteum in patients with femoral hernia using computer tomography and Raman spectroscopy analysis. It was shown that thinning of the periosteum due to atrophy is accompanied by a significant change in the hydroxyapatite $\nu_{1} \mathrm{PO}_{4}$ and Amide I (959/1660 $\left.\mathrm{cm}^{-1}\right)$ Raman peaks ratio and decrease in the $1070 / 959 \mathrm{~cm}^{-1}$ peaks. It was found that Raman spectroscopy is able to confirm the preservation and atrophy degree of the pubic periosteum during surgical resection with an 83\% accuracy. (C) 2021 Journal of Biomedical Photonics \& Engineering.
\end{abstract}

Keywords: periosteum; visualization; X-ray method; computer tomography; ultrasound; Raman spectroscopy.

Paper \#3439 received 21 Jun 2021; revised manuscript received 7 Sep 2021; accepted for publication 7 Sep 2021; published online 2 Nov 2021. doi: 10.18287/JBPE21.07.040502.

\section{Introduction}

Almost all bone structures, with the exception of their intra-articular surfaces, are covered with periosteum, a membrane consisting of the outer fibrous layer and the inner layer (cambium) [1]. Periosteum is important for blood supply to the bone and its regeneration after injury. The periosteal thickening and elevation over the bone may be caused by the development of inflammation or tumor that can be detected by the X-ray method [2]. Periosteum can be detected using magnetic resonance imaging (MRI) but in adults it is not normally visualized separately from the cortical bone layer due to its very low signal [3]. Moreover, it is practically impossible to detect pubic periosteum using computer tomography (CT) if there are no inflammatory changes in the bone. Although the ultrasound method has limitations for bone disease detection it allows imaging the periosteal reaction earlier than by means of conventional radiography. In children, the normal periosteum is visualized as a thin hypoechoic band located above the bone cortex, but in healthy adults it is barely visible on ultrasound scan [4-6].

In 2020, we detected a case [7] of a patient's pubic periosteum completely atrophied due to long-term hernia. The traditional plastic surgery for patients with femoral hernia is only applicable when the Cooper ligament of the pubic periosteum is preserved. Further clinical observations showed the similar periosteum changes in 11 patients. Taking into account the registered clinical data for patients with long-term femoral hernia it was necessary to diagnose pubic periosteum atrophy before and during surgical operation. Ultrasonography is one of the most effective instruments for examining inguinal areas because it is able to confirm the diagnosis of femoral hernia as well as to determine the tactics and the scope of the surgical operation. At the same time, it is problematic to obtain the effective imaging of pubic periosteum by ultrasound even in combination with $\mathrm{X}$ - ray, MRI, and CT when there is no inflammation or no periosteum peeling from the bone. The main problem is the thinning and destruction of periosteum. As a result, only the cortical bone plate is visualized using the ultrasound method while periosteum becomes completely invisible, though this does not mean it is not present. Therefore, other approaches are required for imaging and determination of atrophy and degradation of the periosteum. 
Currently, fluorescence spectroscopy is widely used to study biological tissues including bone tissue [8]. Taking into account the four vascular systems that supply the periosteum (the intrinsic periosteal system located between the fibrous layer and the proliferative layer; the periosteocortical system comprising the nutritional arteries of the periosteum; the musculoperiosteal system responsible for the callus formation after fractures; and the fascioperiosteal system) [9], the periosteum degradation should be accompanied by changes in the endosteal and the periosteal blood flows, as well as in oxidative metabolism. These processes can be detected by the changes in NADH fluorescence [10], but NADH emission spectra are partially overlapped with collagen fluorescence. Moreover, periosteal atrophy causes a decrease in collagen production. These facts make it difficult to analyze periosteum degradation by means of fluorescence spectra. Raman spectroscopy has been reported as an effective method to study biological tissue of different origins including bone tissues, their mineralization, ageing and composition $[11,12]$. The Raman shifts are important contrast indicators of tissue composition, for example, to assess carbonate substitutions for phosphate positions in bone [13, 14]. The bone extracellular matrix includes protein-rich vibrational modes corresponding mainly to collagen properties such as amide backbone, a secondary structure of the protein. However, the absolute Raman intensity is rarely used to analyze tissue properties because they are influenced by the efficiency of Raman scattering and various optical effects (refractive index, specimen surface roughness, etc.). Therefore, many studies apply Raman analysis for the ratios of the maximum peak intensities and the areas under the peaks, for instance, the bone mineralization degree can be defined as the ratio of the hydroxyapatite band $v_{1} \mathrm{PO}_{4}\left(959 \mathrm{~cm}^{-1}\right)$ and Amide I $\left(1640-1720 \mathrm{~cm}^{-1}\right)$ intensities [15]. The other techniques include relative indexes that determine the B-type carbonate substitution $\left(\mathrm{CO}_{3} / \mathrm{v}_{1} \mathrm{PO}_{4}\right)$; overlapping peaks of the ${ }_{2} \mathrm{PO}_{4}$ carbonate vibration and the $\mathrm{v}_{1} \mathrm{CO}_{3}$ vibrations within the $1070 \mathrm{~cm}^{-1}$ region; bending $\mathrm{CH}_{2}$ vibrations within the $1450 \mathrm{~cm}^{-1}$ region; collagen crosslinking (the ratio of the Amide I sub-bands at $1670 \mathrm{~cm}^{-1}$ and $1690 \mathrm{~cm}^{-1}$ ) [16], helical collagen status (the ratio of Amide I sub-bands at $1670 \mathrm{~cm}^{-1}$ and $1640 \mathrm{~cm}^{-1}$ ) are assessed [13]. In general, it is arguable that the whole set of Raman peaks in the $800-1800 \mathrm{~cm}^{-1}$ "fingerprint" region gives a detailed picture of the test tissue, its changes during the disease growth [17, 18], ageing [17], and drug therapy [18]. However, the possibility of Raman spectroscopy to assess the periosteal atrophy degree requires a special study. Therefore, the aim of this work is to establish a possibility of visualization and determination of thinning and atrophy of the pubic periosteum all the way until its complete degradation in patients with femoral hernia.

\section{Materials and Methods}

X-ray, CT, and ultrasonographic methods as well as the Raman spectroscopy were carried out for imaging and detecting pubic periosteum in patients with femoral hernia. The X-ray, ultrasound, and CT data of the Lonny bones in patients with femoral and groin hernias were compared with the opposite side without hernia.

Raman spectroscopy was applied for the ex vivo bone samples taken during autopsies to identify preservation or atrophy of the femoral canal pubic periosteum. The periosteum was removed from a part of the pubic bone samples by an edge-tool, and then the ex vivo samples were delivered to the laboratory for spectral analysis. To ensure the objectivity of the study, the researcher of the optical laboratory did not know on what bone samples the periosteum was preserved.

The ex vivo study of the bone samples was performed using spectroscopic system that allows for simultaneous Raman and fluorescence signal registration in the nearinfrared region [19]. The experimental setup included a thermally stabilized LML-785.0RB-04 laser diode module $(785 \pm 0.1 \mathrm{~nm}$ central wavelength, NECSEL, USA), a QE65Pro portable spectrometer (Ocean Optics Inc., USA) with a matrix CCD detector cooled down to $15^{\circ} \mathrm{C}$, an optical Raman probe (inPhotonics, USA). Laser radiation was delivered to the optical probe using the exciting fiber (100 $\mu \mathrm{m}$ diameter, $0.22 \mathrm{NA})$ and a collimating lens. The band-pass filter cuts out noise and fiber illumination. The dichroic mirror transmits the $785 \mathrm{~nm}$ radiation on the lens that focuses the excitation wavelength on the bone surface. The same lens collects the backscattering light that includes the Raman and the fluorescence signals from the irradiated bone area. The dichroic mirror directs the signal to the recording channel, that includes a long-wavelength filter for filtering the exciting $785 \mathrm{~nm}$ radiation and then to the corresponding lenses and the collecting optical fiber (200 $\mu \mathrm{m}$ diameter, $0.22 \mathrm{NA}$ ) connected to the spectrometer. A detailed description of the experimental setup is given in Ref. [19]. The bone and the periosteum fluorescence spectra were registered within the $800-914 \mathrm{~nm}$ spectral region at the acquisition time of $10 \mathrm{sec}$ with triple accumulation. The laser power on the distal fiber of the optical probe was $150 \mathrm{~mW}$.

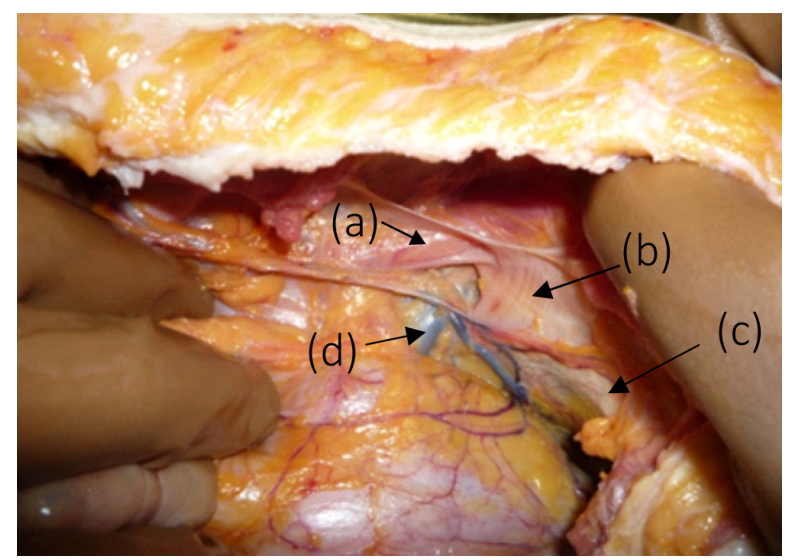

Fig. 1 The left femoral canal area from the abdominal cavity side: (a) Poupart ligament; (b) lunar (Zhimbernatov) ligament; (c) pubic bone covered with periosteum; (d) femoral vein. 


\section{Results and Discussion}

Normally, the femoral canal is absent, but from the side of the abdominal cavity, a slit-like space between the medial wall of the femoral vein, the Poupart and the semilunar ligaments, and the pubic bone covered with the periosteum is clearly observed (Fig. 1). The slit-like space expands and a hernial sac exits through it during the femoral canal formation, which can cause atrophy of the pubic periosteum during a prolonged asymptomatic disease due to permanent friction of the hernial sac on the periosteum. The use of traditional plastic surgery methods is not possible for the femoral canal because of atrophy and thinning of the pubic periosteum.

The X-ray (Fig. 2) and the CT (Fig. 3) images of the pelvis of adult patients in the pubic articulation area clearly show a compact bone plate, but the periosteum is not distinctly visualized even in the case of a traumatic lesion. A compact substance can be clearly defined along the fracture line in the pubic articulation area in Fig. 3 but the periosteum is not observed.

The ultrasound study (Fig. 4 (a, b)) of 18-20-year-old patients revealed that the periosteum is determined as a hypoechoic band covering the anterior iliac cortex. The ultrasound scan of the spoke-bone in a 35-year-old patient shows the same result (Fig. 4 (c)), whereas for a 40-year-old patient the ultrasound transverse section clearly shows (Fig. $4(\mathrm{~d})$ ) the superior pubic bone (asterisk) and an unchanged cortical layer of the bone is observed as a normal hyperechoic line (arrow) with a posterior acoustic shielding.

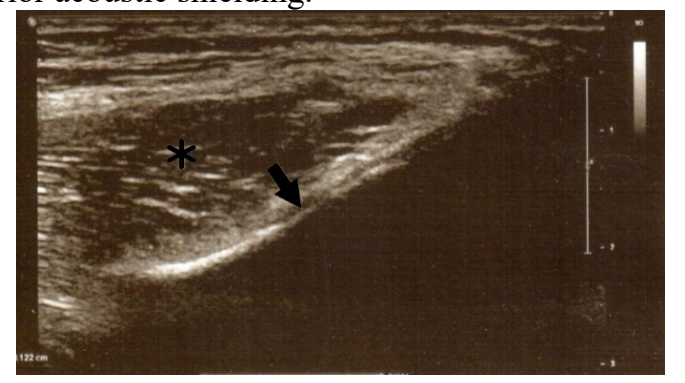

(a) 18-year-old patient

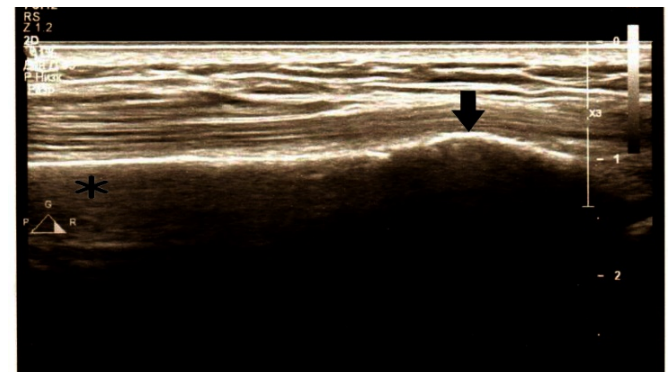

(c) 35-year-old patient

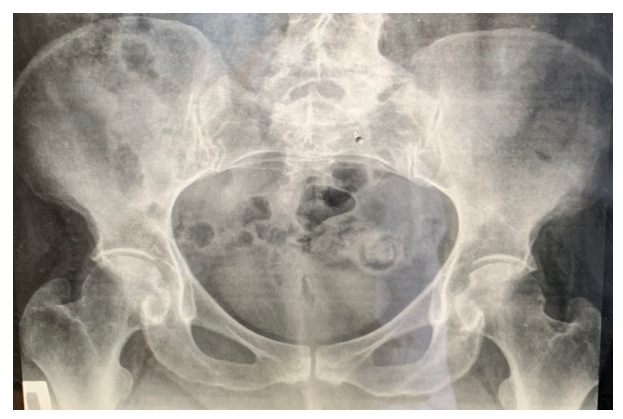

Fig. 2 X-ray image of the patient pelvis after the surgical resection of the hernia on the right. The compact bone substance is clearly visualized in the pubic articulation area.

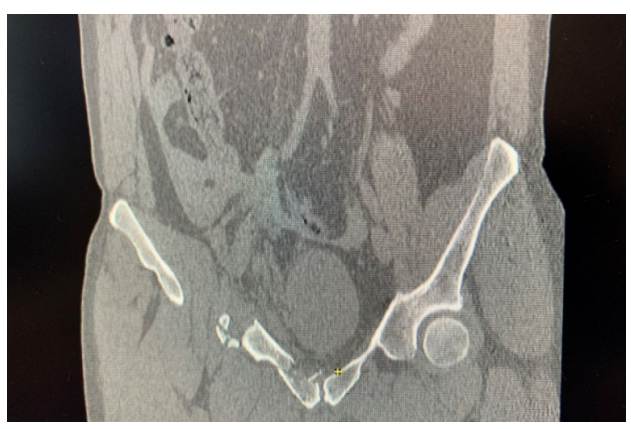

Fig. 3 CT image in the frontal plane of the pelvis with a pubic bone fracture.

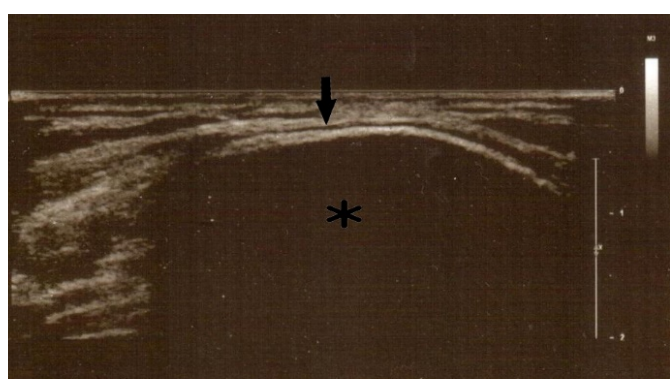

(b) 20-year-old patient

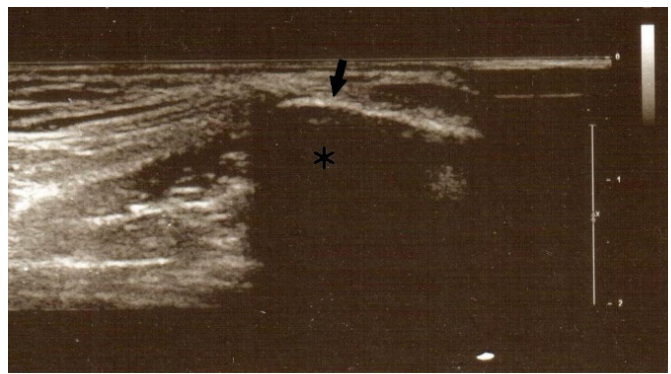

(d) 40-year-old patient

Fig. 4 The ultrasound scanning of the pubic bone: (a) on the longitudinal sonogram, the periosteum (arrow) is visualized as a hypoechoic strip covering the anterior part of the iliac cortex (asterisk); (b) the longitudinal section of the sonogram shows the anterior superior iliac spine (asterisk), thin periosteum is visualized as a hypoechoic strip (arrow); (c) the sonogram shows a longitudinal section of the upper third of the radius (asterisk), the hyperechoic line (arrow) corresponds to the cortical layer of the bone; (d) the transverse section of the anterior iliac spine (asterisk). On the ultrasound scanning, the unaltered cortical layer of the bone is observed as a normal hyperechoic line (arrow) with posterior acoustic dimming and some reverberation (artifacts). 
The ultrasound scanning of a femoral hernia is shown in Fig. 5, where the arrow indicates the cortical layer of the bone (hyperechoic continuous line) and the rhombus marks the hernia area. The Doppler signal is not recorded, but the artery feeding the bone is visible.

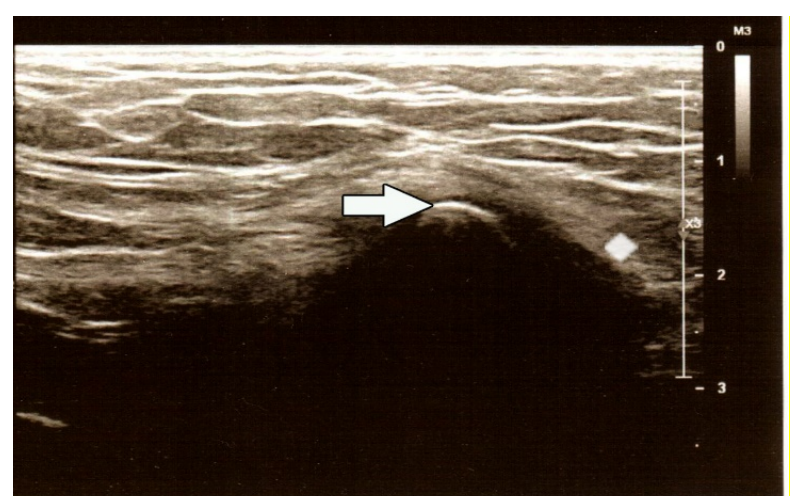

Fig. 5 The echogram of a longitudinal section of the superior iliac spine. The hyperechoic continuous line corresponds to the cortical bone (arrow). The rhombus indicates the femoral hernia.

The obtained results correlate with the study by A. D. Moraux et al. [6] stating that periosteum is reliably indicated on ultrasound images only for children, while in older age persons its determination is difficult. Other studies $[4,6]$ revealed that ultrasound scanning demonstrates a periosteal response indicating bone disease earlier than conventional X-rays. Considering that femoral hernia mainly affects the elderly, it is practically impossible to determine the atrophy degree, thinning or complete degradation of the pubic periosteum before surgery. Therefore, it is necessary to develop some intra-operative techniques for express analysis of the state of the periosteum pubic bone for which Raman spectroscopy seems to be one of the most promising methods.

Four pubic bone samples were studied ex vivo using spectral analysis. The fluorescence and the Raman spectra in the 800-914 $\mathrm{nm}$ band were measured from the random points at the central area of the bone and the periosteum surfaces (see Fig. 6) to avoid possible distortions associated with edge effects (residual mechanical tension or exfoliation of surface layers).

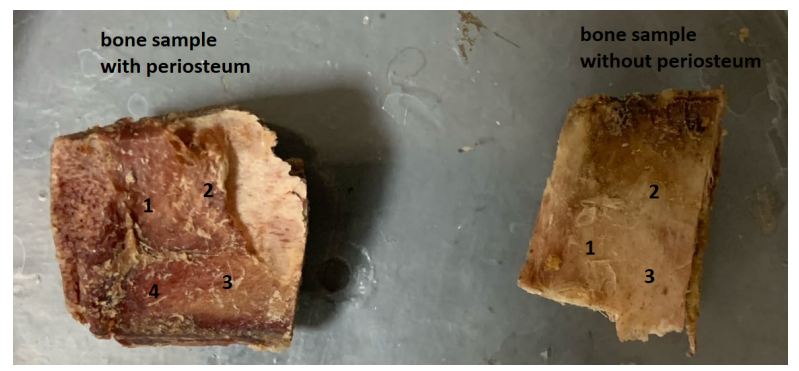

Fig. 6 Photo of the studied bone samples.

Fig. 7 shows the periosteum (blue) and bones (red) fluorescence spectra smoothed by the Savitzky-Golay filter and normalized to the standard deviation. The fluorescence spectrum is the falling curve with maximal value at the $800-810 \mathrm{~nm}$ initial region without highlighted spectral signatures. The fluorescence curve and its first derivative do not include any significant spectral differences, which could be associated with the presence or absence of the periosteum in the studied samples. Apparently, it can be a result of a large contribution of the bone substrate to the fluorescence signal that masks the fluorescence changes associated with the presence of a periosteum thin layer (less than $1 \mathrm{~mm})$.

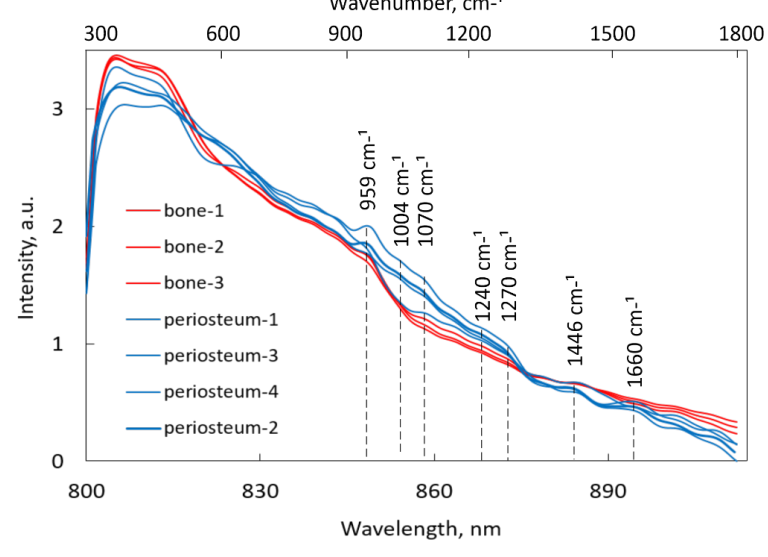

Fig. 7 The fluorescence spectra of periosteum and bone.

The bone Raman features are observed in the 900-1800 $\mathrm{cm}^{-1}$ wavenumber region. Therefore, to analyze the bone Raman spectrum, the fluorescence background is mathematically subtracted from the registered spectrum in $844-914 \mathrm{~nm}$ spectral region by a $5^{\text {th }}$ order polynomial approximation (Fig. 8a) [20, 21]. Fig. $8 \mathrm{~b}$ demonstrates the Raman spectra of the bone and the periosteum samples normalized to the standard deviation after fluorescence removal.

The most prominent Raman band in both the periosteum and the bone spectra is observed at the $959 \mathrm{~cm}^{-1}$ (symmetric stretching $\mathrm{PO}_{4}{ }^{3-}$ vibrations), which is associated with the contribution of the phosphate mineral. The narrow Raman band at $1004 \mathrm{~cm}^{-1}$ (phenylalanine) is merging with the $959 \mathrm{~cm}^{-1}$ band forming its shoulder peak. The highlighted Raman peak of the B-type carbonate at $1070 \mathrm{~cm}^{-1}\left(v_{1} \mathrm{CO}_{3}{ }^{2-}\right)$ is expanded due to overlapping with a weaker peak at the $1076 \mathrm{~cm}^{-1}\left(\mathrm{v}_{3} \mathrm{PO}_{4}{ }^{3-}\right)$ caused by phosphate vibrations. The subtraction of the fluorescence background is able to detect the weak wide Raman peaks with the central maxima: a double 1240-1280 $\mathrm{cm}^{-1}$ band (Amide III), within the $1440-1450 \mathrm{~cm}^{-1}$ area (with the maximum at $1446 \mathrm{~cm}^{-1}$ associated with scissoring vibrations of the $\mathrm{CH}_{2}$ in lipids), at $1640-1678 \mathrm{~cm}^{-1}$ (Amide I, with the maximum at the $1665 \mathrm{~cm}^{-1}$ corresponding to the polarized stretching vibrations $\mathrm{C}=\mathrm{O}$ ). Localization of the sub-peaks in the Amide I and Amide III bands was determined by the local minima of the second derivative of the spectrum therefore it was fractionally varied for 


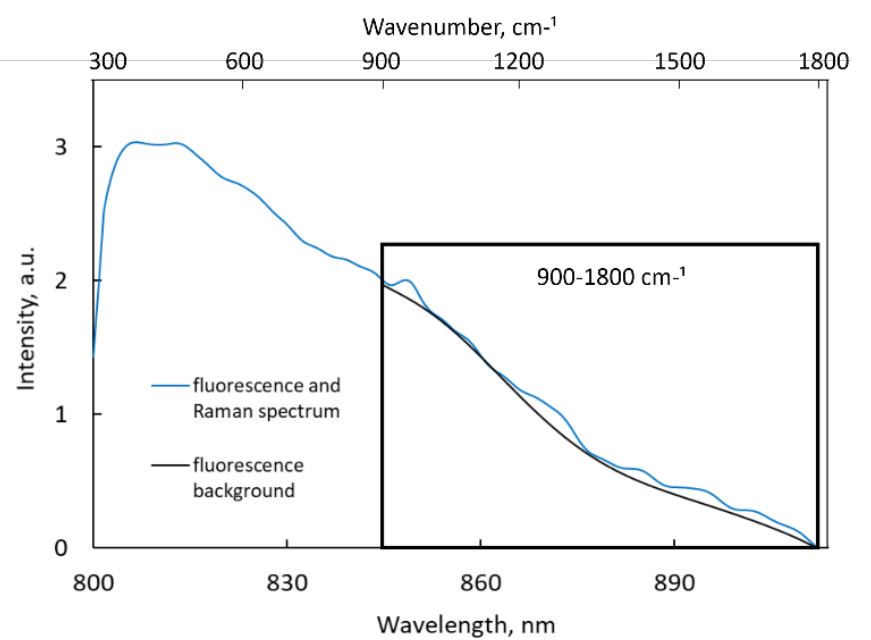

(a)

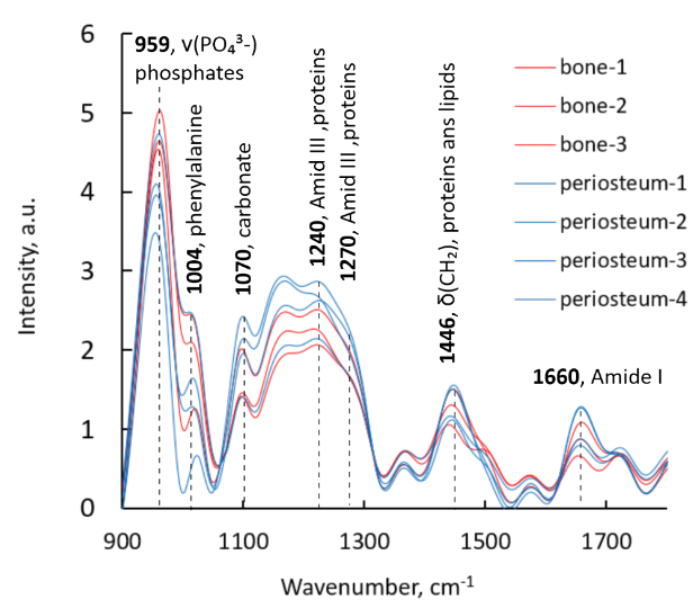

(b)

Fig. 8 (a) fluorescence background in the analyzed 900-1800 $\mathrm{cm}^{-1}$ Raman area; (b) the normalized Raman spectra of the periosteum and bone after background removal.

Table 1 Raman indexes of the bone and periosteum samples.

\begin{tabular}{cccc} 
& \multicolumn{2}{c}{ Mean value (Standard deviation) Scatter } & \multirow{2}{*}{ ROC AUC } \\
\cline { 2 - 3 } Indexes* & Bone $(\mathbf{n}=\mathbf{3})$ & Periosteum $(\mathbf{n}=\mathbf{4})$ & 0.75 \\
\hline $\mathrm{A}_{1070} / \mathrm{A}_{959}$ & $0.35(0.08) 0.16$ & $0.48(0.17) 0.22$ & 0.50 \\
\hline $\mathrm{A}_{1070} / \mathrm{A}_{1660}$ & $1.32(0.13) 0.26$ & $1.35(0.23) 0.52$ & 0.75 \\
\hline $\mathrm{A}_{959} / \mathrm{A}_{660}$ & $3.86(0.82) 1.63$ & $2.98(0.94) 2.2$ & 0.83 \\
\hline $\mathrm{I}_{1070} / \mathrm{I}_{959}$ & $0.37(0.07) 0.13$ & $0.51(0.13) 0.31$ & 0.50 \\
\hline $\mathrm{I}_{1070} / \mathrm{I}_{1660}$ & $1.6(0.15) 0.29$ & $1.65(0.24) 0.54$ & 0.83 \\
\hline $\mathrm{I}_{959} / \mathrm{I}_{1660}$ & $4.39(0.67) 1.32$ & $3.4(0.85) 1.9$ & 0.75 \\
\hline $\mathrm{I}_{1240} / \mathrm{I}_{1446}$ & $1.64(0.21) 0.38$ & $1.7(0.19) 0.46$ & 0.67 \\
\hline $\mathrm{I}_{1660} / \mathrm{I}_{1240}$ & $0.50(0.01) 0.016$ & $0.47(0.046) 0.09$ & \\
\hline$* \mathrm{I}-$ ratio of the maximum intensities of the Raman peaks; & & \\
\hline $\begin{array}{l}\mathrm{A}-\text { integral indexes of the area under the peaks; } \\
\text { subscript indicates the peak position }\left(\mathrm{cm}^{-1}\right)\end{array}$ & & \\
\hline
\end{tabular}

different samples. It should be noted that the Raman peaks at the $959 \mathrm{~cm}^{-1}$ correspond to the mineral contribution (phosphate) while the peaks at 1240-1280, 1445, $1665 \mathrm{~cm}^{-1}$ correspond to the collagen contributions. Taking into account the small cohort of bone samples (3 spectra of the bone, 4 spectra of periosteum), to identify periosteum, spectral indexes of mineralization, carbonation, and the ratio of the organic and the mineral phases were calculated. The spectral index represents the ratio of the maximum intensities (I) or the areas under the peaks (A) at the Raman bands in question for each spectrum. Table 1 and Fig. 9 demonstrate the calculated Raman indexes and their graphical comparison for bones and periosteum samples.
Comparison of the Raman indexes has proved that for bone tissue analysis, the most informative is the ratio of the $v_{1} \mathrm{PO}_{4}{ }^{3-} /$ Amide I $\left(\mathrm{I}_{95} / \mathrm{I}_{1660}\right)$ and ${ }_{1} \mathrm{CO}_{3}{ }^{2} / v_{1} \mathrm{PO}_{4}{ }^{3-}$ ( $\left.\mathrm{I}_{1070} / \mathrm{I}_{959}\right)$ : the mean values of these indexes significantly differ between the bone and the periosteum spectra, and their dispersions scarcely overlap. For differentiating the bone and the periosteum samples on the basis of the $\mathrm{I}_{959} / \mathrm{I}_{1660}$ and $\mathrm{I}_{1070} / \mathrm{I}_{959}$ indexes, the ROC AUC is equal to 0.83 . The mean value of the $\mathrm{I}_{959} / \mathrm{I}_{1660}$ mineralization indexes for the bone spectra is 1.3 times higher in comparison with the periosteum, and the $\mathrm{I}_{1070 / \mathrm{I}_{959}}$ carbonate-phosphate index is 1.4 times lower, respectively. Therefore, analysis of these indexes allows detecting the progressive degradation and atrophy of the 


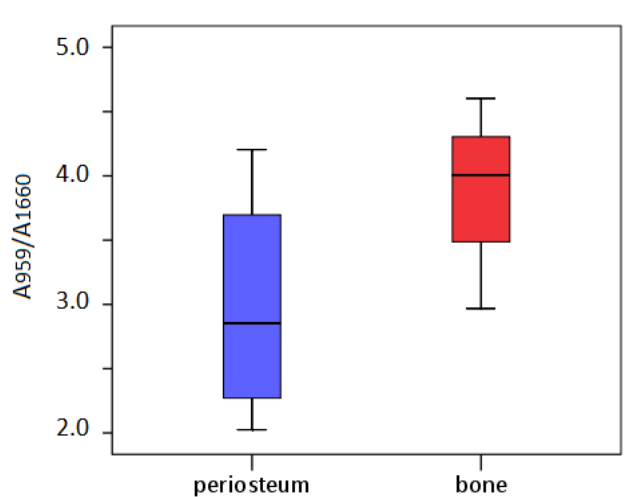

(a)

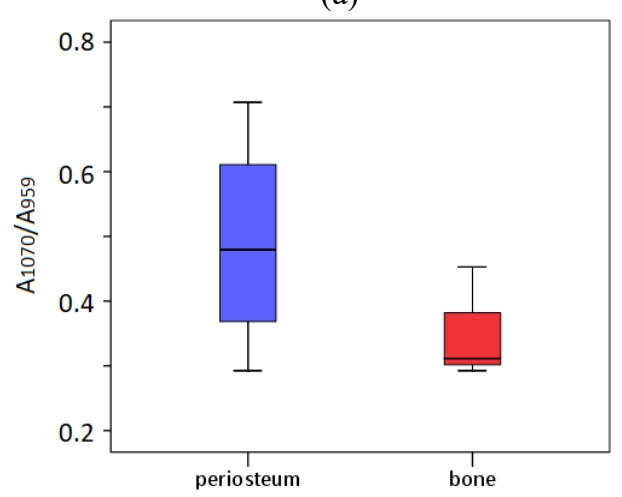

(c)

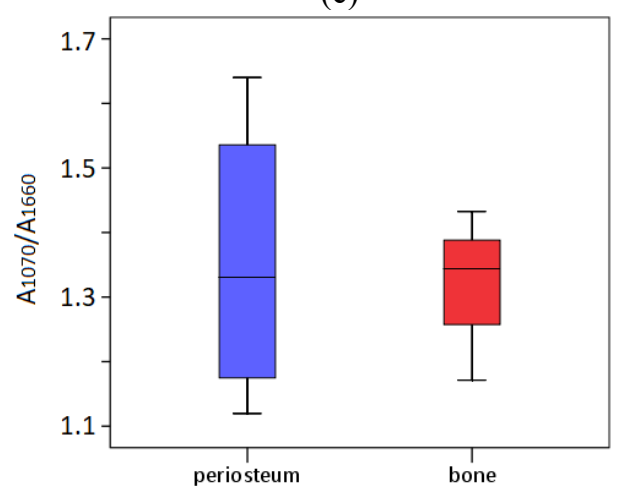

(e)

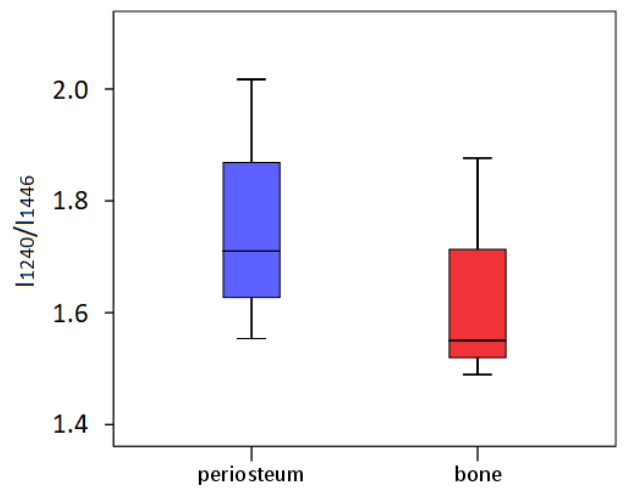

(g)

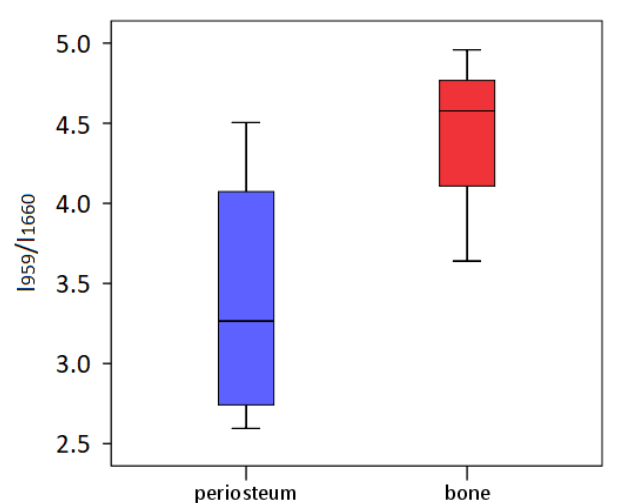

(b)

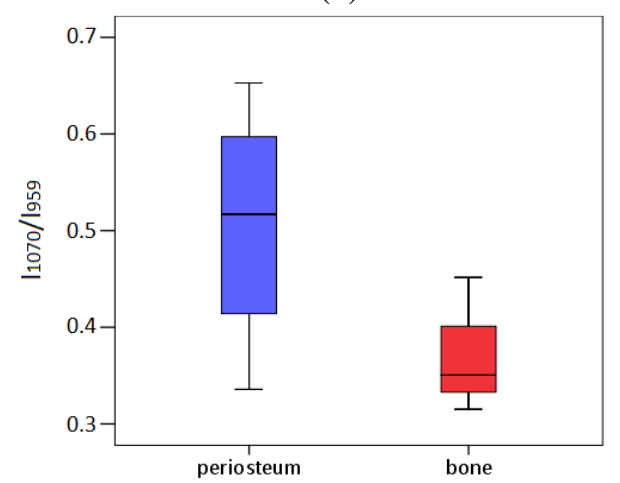

(d)

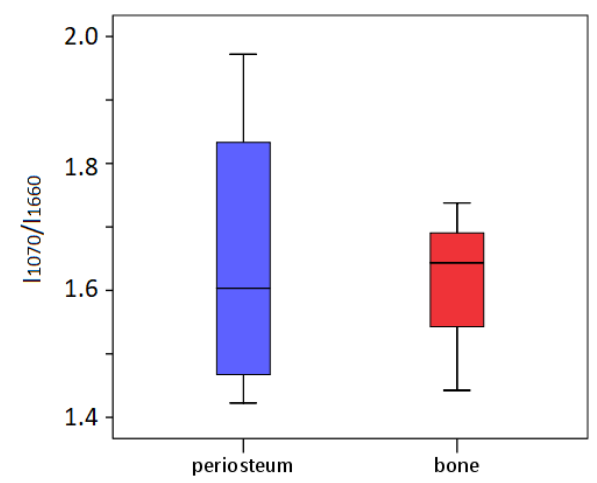

(f)

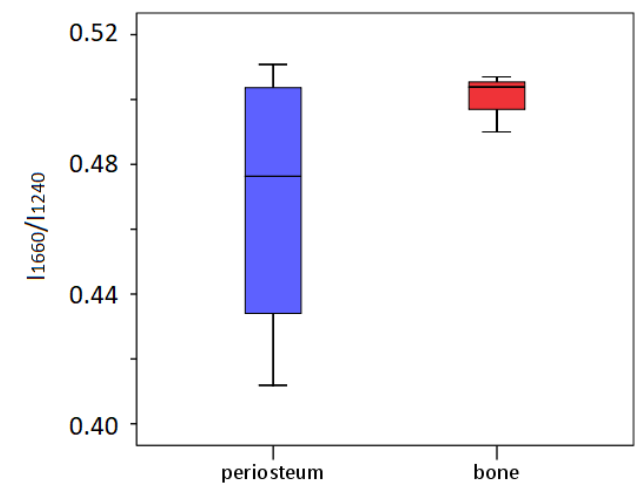

(h)

Fig. 9 Box plot diagrams with a mean value and standard deviation of the Raman indexes for periosteum (blue) and bone (red) samples, where (a) is the area ratio and (b) is the intensity ratio for the $v_{1} \mathrm{PO}_{4}{ }^{3-}\left(959 \mathrm{~cm}^{-1}\right)$ and $\mathrm{Amid} \mathrm{I}\left(1660 \mathrm{~cm}^{-1}\right)$ peaks; (c) is the area ratio and (d) is the intensity ratio for the carbonate $v_{1} \mathrm{CO}_{3}{ }^{2-}\left(1070 \mathrm{~cm}^{-1}\right)$ and phosphate $v_{1} \mathrm{PO}_{4}{ }^{3-}$ $\left(959 \mathrm{~cm}^{-1}\right)$ peaks; (e) is the area ratio and (f) is the intensity ratio for the carbonate $v_{1} \mathrm{CO}_{3}{ }^{2-}\left(1070 \mathrm{~cm}^{-1}\right)$ and Amide I $\left(1660 \mathrm{~cm}^{-1}\right)$ peaks. For the Amide I $\left(1660 \mathrm{~cm}^{-1}\right)$ and Amide III $\left(1240 \mathrm{~cm}^{-1}\right)$ ratios as well as for the Amide III $\left(1240 \mathrm{~cm}^{-1}\right)$ and $v\left(\mathrm{CH}_{2}\right)$ ratio $\left(1446 \mathrm{~cm}^{-1}\right)$ in lipids, only the intensity ratios are shown $(\mathrm{g})$ and $(\mathrm{h})$ respectively). 
periosteum and the manifestation of the bone spectral features in the Raman spectrum. Furthermore, it was observed that the indexes of the Amide I / Amide III $\left(\mathrm{I}_{1660} / \mathrm{I}_{1240}\right)$ and $\mathrm{I}_{1240} / \mathrm{I}_{1446}$ organic phases that characterize the ratio between the proteins and lipids concentrations are changing along with the change of mineralization and carbonization parameters. The changes of the organic parameters are less pronounced but in combination with the mineralization indexes they can be associated with a degree of periosteum atrophy. However, to prove this suggestion, it is necessary to perform clinical study with a large cohort of bone samples.

Comparison of the Raman indexes has proved that for bone tissue analysis, the most informative is the ratio of the $v_{1} \mathrm{PO}_{4}{ }^{3-} /$ Amide I $\left(\mathrm{I}_{959} / \mathrm{I}_{1660}\right)$ and $v_{1} \mathrm{CO}_{3}{ }^{2} / v_{1} \mathrm{PO}_{4}{ }^{3-}$ $\left(\mathrm{I}_{1070} / \mathrm{I}_{959}\right)$ : the mean values of these indexes significantly differ between the bone and the periosteum spectra, and their dispersions scarcely overlap. For differentiating the bone and the periosteum samples on the basis of the $\mathrm{I}_{959} / \mathrm{I}_{1660}$ and $\mathrm{I}_{1070} / \mathrm{I}_{959}$ indexes, the ROC AUC is equal to 0.83 . The mean value of the $\mathrm{I}_{959} / \mathrm{I}_{1660}$ mineralization indexes for the bone spectra is 1.3 times higher in comparison with the periosteum, and the $\mathrm{I}_{1070} / \mathrm{I}_{959}$ carbonate-phosphate index is 1.4 times lower, respectively. Therefore, analysis of these indexes allows detecting the progressive degradation and atrophy of the periosteum and the manifestation of the bone spectral features in the Raman spectrum. Furthermore, it was observed that the indexes of the Amide I / Amide III $\left(\mathrm{I}_{1660} / \mathrm{I}_{1240}\right)$ and $\mathrm{I}_{1240} / \mathrm{I}_{1446}$ organic phases that characterize the ratio between the proteins and lipids concentrations are changing along with the change of mineralization and carbonization parameters. The changes of the organic parameters are less pronounced but in combination with the mineralization indexes they can be associated with a degree of periosteum atrophy. However, to prove this suggestion, it is necessary to perform clinical study with a large cohort of bone samples.

\section{Conclusion}

The periosteal atrophy was studied using X-ray, ultrasound, computer tomography, and optical spectroscopy. Using the X-ray method and CT before surgery is not sufficient to establish the preservation or atrophy of the pubic periosteum in the femoral canal area. With ultrasound scanning, visualization of the pubic periosteum is only possible for young patients. The spectroscopy method makes it possible to identify the degradation / atrophy of the pubic periosteum (with an accuracy of at least $83 \%$ ) during intra-operative express analysis. The time of the spectral study (spectrum registration, mathematical processing of spectra and determination of Raman parameters) does not exceed 10-20 sec. However, additional clinical studies are needed to identify the peculiarities relations between Raman parameters and the age, gender, and the degree of periosteal atrophy.

\section{Disclosures}

All authors declare that there is no conflict of interests in this paper.

\section{References}

1. J. R. Dwek, "The periosteum: what is it, where is it, and what mimics it in its absence?" Skeletal radiology 39(4), 319-323 (2010).

2. D. Bisseret, R. Kaci, M. H. Lafage-Proust, M. Alison, C. Parlier-Cuau, J.-D. Laredo, and V. Bousson, "Periosteum: Characteristic imaging findings with emphasis on radiologic-pathologic comparisons," Skeletal radiology 44, 321338 (2015).

3. I. L. Reichert, M. Benjamin, P. D. Gatehouse, K. E. Chappell, J. Holmes, T. He, and G. M. Bydder, "Magnetic Resonance Imaging of Periosteum With Ultrashort TE Pulse Sequences,” Journal of Magnetic Resonance Imaging 19(1), 99-107 (2004).

4. R. S. Rana, J. S. Wu, and R. L. Eisenberg, "Periosteal reaction,” American Journal of Roentgenology 193(4), W259W272 (2009).

5. D. F. Hoffman, E. Adams, and S. Bianchi, "Ultrasonography of fractures in sports medicine," British Journal of Sports Medicine 49(3), 152-160 (2015).

6. A. D. Moraux, S. Gitto, and S. Bianchi, "Ultrasound Features of the Normal and Pathologic Periosteum," Journal of Ultrasound in Medicine 38(3), 775-784 (2019).

7. V. I. Belokonev, A. G. Zharov, "Features of Surgical Treatment of Patients with Uncomplicated Femoral Hernia," Novosti Khirurgii 27(2), 146-152 (2019) [in Russian].

8. D. Frikha-Benayed, J. Basta-Pljakic, R. J. Majeska, and M. B. Schaffler, "Regional differences in oxidative metabolism and mitochondrial activity among cortical bone osteocytes," Bone 90, 15-22 (2016).

9. C. H. M. F. Alencar, C. R. S. Silveira, M. M. Cavalcante, C. G. M. Vieira, M. J. D. Teixeira, F. A. Neto, A. de Abreu, and A. Chhabra, "Periosteum: An imaging review," European Journal of Radiology Open 7, 100249 (2020).

10. S. Jiang, J. T. Elliott, J. R. Gunn, C. Xu, A. J. Ruiz, B. W. Pogue, E. R. Henderson, and I. L. Gitajn, "Endosteal and periosteal blood flow quantified with dynamic contrast-enhanced fluorescence to guide open orthopaedic surgery," Proceedings of SPIE 11222, 112220F (2020). 
11. V. P. Zakharov, I. A. Bratchenko, D. N. Artemyev, O. O. Myakinin, S. V. Kozlov, A. A. Moryatov, and A. E. Orlov, "Multimodal Optical Biopsy and Imaging of Skin Cancer," Chapter 17 in Neurophotonics and Biomedical Spectroscopy, R. R. Alfano, L. Shi (Eds.), Elsevier, 449-476 (2019). ISBN: 978-0-323-48067-3.

12. E. Brauchle, D. C. Berrio, M. Rieger, K. Schenke-Layland, S. Reinert, and D. Alexander, "Raman Spectroscopic Analyses of Jaw Periosteal Cell Mineralization,” Stem Cells International 2017, 1651376 (2017).

13. M. Unal, S. Uppuganti, S. Timur, A. Mahadevan-Jansen, O. Akkus, and J. S. Nyman, “Assessing matrix quality by Raman spectroscopy helps predict fracture toughness of human cortical bone," Scientific Reports 9, 7195 (2019).

14. M. D Morris, G. S. Mandair, "Raman assessment of bone quality," Clinical Orthopaedics and Related Research 469(8), 2160-2169 (2011).

15. M. Fraulob, S. Pang, S. L. Cann, R. Vayron, M. Laurent-Brocq, S. Todatry, J. A. N. T. Soares, I. Jasiuk, and G. Haïat, "Multimodal characterization of the bone-implant interface using Raman spectroscopy and nanoindentation," Medical Engineering and Physics 84, 60-67 (2020).

16. E. Paschalis, K. Verdelis, S. B. Doty, A. L. Boskey, R. Mendelsohn, and M. I. Y. M. Yamauchi, "Spectroscopic Characterization of Collagen Cross-Links in Bone," Journal of Bone and Mineral Research 16(10), 1821-1828 (2001).

17. E. Paschalis, S. Gamsjaeger, and K. Klaushofer, "Vibrational spectroscopic techniques to assess bone quality," Osteoporosis International 28(8), 2275-2291 (2017).

18. G. S. Mandair, M. D. Morris, "Contributions of Raman spectroscopy to the understanding of bone strength," BoneKEy Reports 4, 620 (2015).

19. I. A. Bratchenko, L. A.Bratchenko, A. A. Moryatov, Y. A. Khristoforova, D. N. Artemyev, O. O. Myakinin, A. E. Orlov, S. V. Kozlov, and V. P. Zakharov, "In vivo diagnosis of skin cancer with a portable Raman spectroscopic device," Experimental Dermatology 30(5), 652-663 (2021).

20. H. Zeng, H. Lui, D. I. McLean, and H. Zeng, “Automated autofluorescence background subtraction algorithm for biomedical Raman spectroscopy," Applied spectroscopy 61(11), 1225-1232 (2007).

21. L. Shamina, I. Bratchenko, D. Artemyev, O. Myakinin, A. Moryatov, O. Kaganov, A. Orlov, S. Kozlov, and V. Zakharov, "Raman and autofluorescence analysis of human body fluids from patients with malignant tumors," Journal of Biomedical Photonics \& Engineering 3(2), 020308 (2017). 